\title{
The Interaction of Heart Rate and Blood Pressure Variations for AV Dissociation Illustrated by a Closed-Loop Heart Rate Control Model
}

\author{
HW Chiu \\ Graduate Institute of Medical Informatics, Taipei Medical University, Taipei, Taiwan
}

\begin{abstract}
The atrial rate variability measured under an atrialventricular $(A V)$ disassociation condition, e.g. fixed-rate ventricular pacing, has shown a pattern in which the main frequency components varied with the ventricular rates that very different with the $A V$ synchronization condition. Some evidences showed that beat-to-beat blood pressure variation might play a role for this phenomenon. To demonstrate the possible mechanism for the interaction between atrial rate variability and blood pressure variability in AV dissociation condition, a closeloop heart rate control model considering the baroreflex was created in this study. In this model, an integrating pulse frequency modulation model was used to generate the heartbeat and its input was transformed from the blood pressure waveform. One simulation is to automatically generate heart rate variability and blood pressure variability. In this case the blood pressure waveform for each beat was shaped using a curve with a variable maximum representing systolic blood pressure that was determined by each $A V$ delay. The other simulation is to generate atrial rate variability using the real blood pressure waveform data recorded from $A V$ block patient. The results demonstrated that the frequency domain features for the real atrial rate variation could be approximated with real blood pressure waveform data by this model when the mean heart rate was tuned.
\end{abstract}

\section{Introduction}

Heart rate (HR) variability (HRV) had been widely used to assess the autonomic nervous activities (ANA) in many different situations including physiologically or pathologically changes. Mostly HRV is measured under an atrial-ventricular (AV) synchronization condition. In this normal condition the frequency power of HRV mainly locate at low-frequency (LF) band (0.04-0.15 Hz) and high-frequency (HF) band $(0.15-0.4 \mathrm{~Hz})$. But in our previous study under an AV disassociation condition, e.g. fixed-rate ventricular pacing, the atrial rate variability $(\mathrm{ARV})$, was measured and showed a pattern in which the main frequency components varied with the ventricular rates [1]. Some studies was reported that ventricular rate and AV-delay have marked influences on ARV [2,3] Moreover, some evidences have shown that beat-to-beat blood pressure (BP) variation (BPV) may play a role for this phenomenon [4,5]. In our later investigation [6], we found that the oscillation frequency of ARV may be an aliasing phenomenon of BP pulse frequency exactly equal to ventricular rate when the mean atrial rate is considered as the sample rate of ARV signal. Hence we suggested that the BP pulse dominate the ARV in AV dissociation situation. Cardiac aliasing phenomena were also observed in other different experiments [7,8] in which the respiration frequency is over $1 / 2$ mean heart rate so that the HF of HRV is not equal to the breathing rate. Although the relationship between the oscillation frequencies of ARV and ventricular pacing rate (or BP pulse rate) could be derived by the cardiac aliasing, how the ARV is regulated by the BPV needs to be revealed. The regulatory effect of blood pressure on heart rate control is known as baroreflex. Most studies use systolic $\mathrm{BP}$ or diastolic BP, instead of the entire BP waveform, to model this control mechanism [9]. However, the information embedded in systolic BP or diastolic BP is not enough to explain the ARV feature in AV dissociation situation. In this study, a model is created to illustrate the frequency features of ARV in response to entire BP waveform to explain the changes of ARV under AV dissociation.

\section{Methods}

\subsection{IPFM model}

Many heart rate control models have been proposed to simulate the heart rate in response to different physiological situations. Among these models, the integrating pulse frequency modulation (IPFM) model was frequently used to simulate the heartbeat generation $[10,11]$. Suppose that the modulation signal is $m(t)$. When it is integrated to reach a threshold $\mathrm{T}$, a heartbeat generates. The IPFM model is mathematically described as follows: 


$$
T=\int_{t_{n}}^{t_{n+1}} m(t) d t
$$

$t_{n}$ and $t_{n+1}$ are the times of two successive heartbeats and the nth heartbeat interval AA[n] is $\left(t_{n+1}-t_{n}\right)$.

Most researches have considered $m(t)$ for the autonomic influences. Many baroreflex studies showed that the blood pressure regulate heart rate with a negative feedback control mechanism. That is the elevated BP reduces the HR and vice versa. Therefore the BP waveform could be considered to transform as the modulation signal of IPFM model. In this model the relation between BP and modulation signal was simplified as a linear negatively proportional equation:

$$
m(t)=-a * B P+b
$$

Here $a$ and $b$ are constants. They can be tuned to fit different situations.

\subsection{Real data simulation}

First an open-loop model was applied. The real BP waveform was transformed to be the modulation signal $\mathrm{m}(\mathrm{t})$ according to equation (2) to generate the simulated ARV. The real ARV and BP data of reference [6] were used to simulate and compare. Because the mean HR of each experiment is different, the constants $\mathrm{a}$ and $\mathrm{b}$ were tuned to generate the ARV with a similar mean HR to real ARV. The spectra of real ARV and simulated ARV were compared.

\subsection{Close-loop model}

In addition to simulate the ARV used the real BP data, a close-loop model was also created to illustrate the interaction between ARV and BPV. Due to AV dissociation the AV delay varies from beat to beat. The irregularly changed AV delays will induce fluctuations of BP waveform. Here we suppose the systolic pressure is the only parameter influenced by AV delay. Thus the blood pressure waveform for each beat was shaped using a curve with a variable maximum representing systolic blood pressure that was determined by each AV delay. The relationship between AV delay and systolic blood pressure (SBP) shows that the longer or shorter AV delay will output a lower systolic pressure. Therefore it was supposed that SBP and AV delay have the following relation:

$$
S B P=60 \times e^{-(\text {AVdelay }-150)^{2}}+60
$$

Thus SBP has an optima value $120 \mathrm{mmHg}$, and gradually decay when AV delay become longer or shorter. Figure 1 shows this close-loop model. The A and $\mathrm{V}$ indicate the time points of atrial and ventricular contraction respectively.

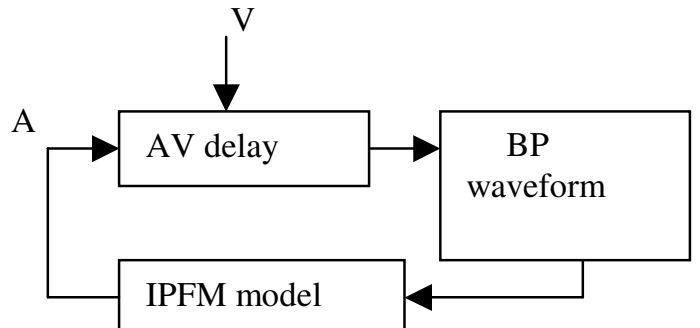

Figure 1. A close-loop model for automatically generating $\mathrm{BP}$ waveform and $\mathrm{ARV}$ in $\mathrm{AV}$ dissociation situation. The effects of AV delay and baroreflex were included.

\section{Results}

\subsection{Real data simulation}

The ARV in fixed-rate ventricular pacing was simulated using real BP waveform data obtained from the non-invasive continuous blood pressure device. Figure 2 shows a simulation result of $120 \mathrm{bpm}$ pacing rate. From top to bottom, the real ARV, the simulated ARV, the power spectrum of the real ARV and the power spectrum of the simulated ARV were plotted.
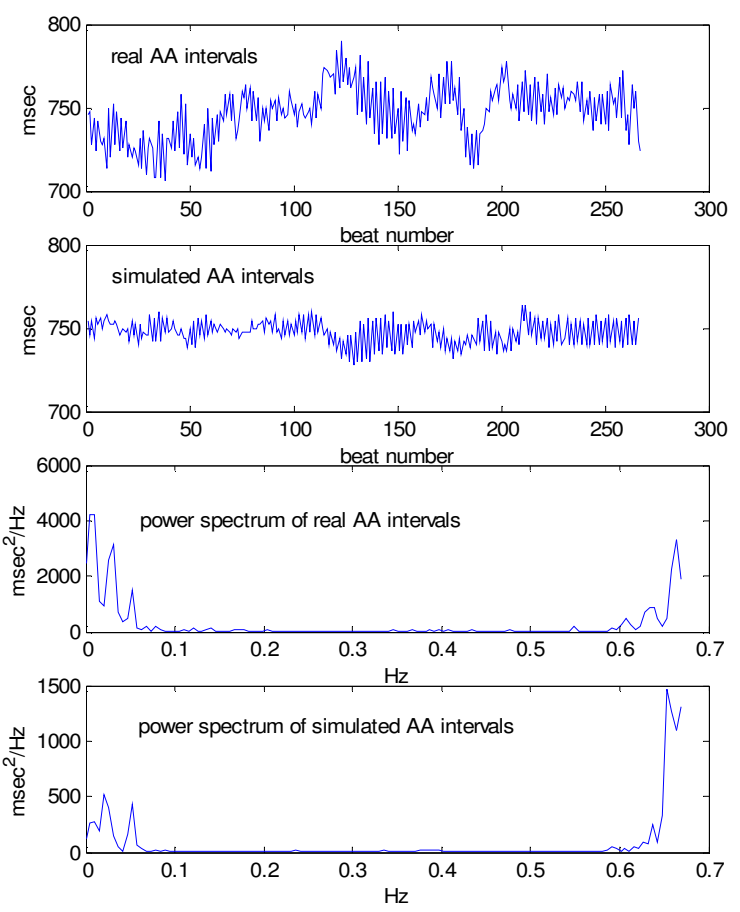

Figure 2. An ARV simulation result of $120 \mathrm{bpm}$ pacing rate using real $\mathrm{BP}$ waveform data. The simulated ARV had the very similar frequency feature with the real ARV.

The power spectrum of the simulated ARV is similar to that of the real ARV. The real mean atrial contraction 
interval (AA interval) extracted from an esophageal lead is $746 \mathrm{~ms}$. The parameter $\mathrm{a}$ and $\mathrm{b}$ in equation (2) were selected as 0.0025 and 1.45 respectively so that the simulated ARV had the same mean atrial rate. The dominant frequencies of ARV are located at about 0.03 $\mathrm{Hz}$ and $0.67 \mathrm{~Hz}$.

Figure 3 shows another simulation result of $90 \mathrm{bpm}$ ventricular pacing rate. The real mean AA interval was $640 \mathrm{~ms}$ and the simulated ARV had a similar mean AA interval by selecting $a$ and $b$ in equation (2) as 0.0025 and 1.73. The spectra of real and simulated ARV are very consistent.
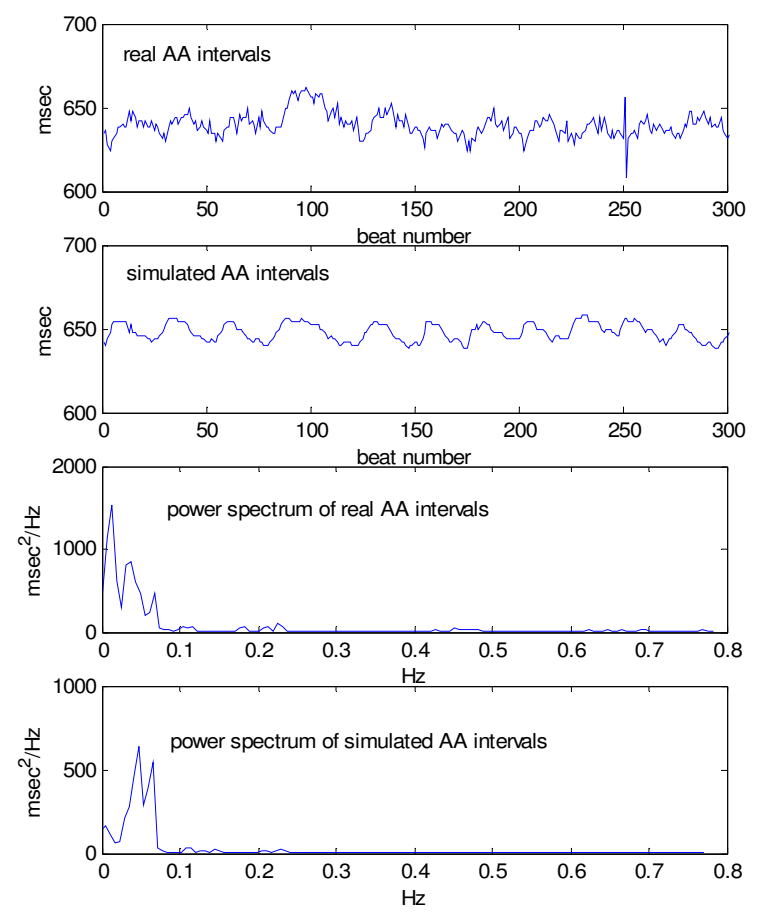

Figure 3. An ARV simulation result of $90 \mathrm{bpm}$ pacing rate using real $\mathrm{BP}$ waveform data. The simulated ARV had the $0.06 \mathrm{~Hz}$ dominant frequency that is similar to the real ARV.

\subsection{Close-loop model simulation}

A simulated ARV and its power spectrum under 90 bpm ventricular pacing was obtained and shown in figure 4. The mean atrial rate of this ARV was $1.22 \mathrm{~Hz}$. According to the cardiac aliasing the dominant frequency of ARV should be $0.28 \mathrm{~Hz}$. The simulation results showed a consistent characteristic. Figure 5 shows another simulation result of $120 \mathrm{bpm}$ ventricular pacing. The mean atrial rate of this ARV was also $1.22 \mathrm{~Hz}$, hence the dominant frequency of ARV should be $0.44 \mathrm{~Hz}$. The consistent result was illustrated.
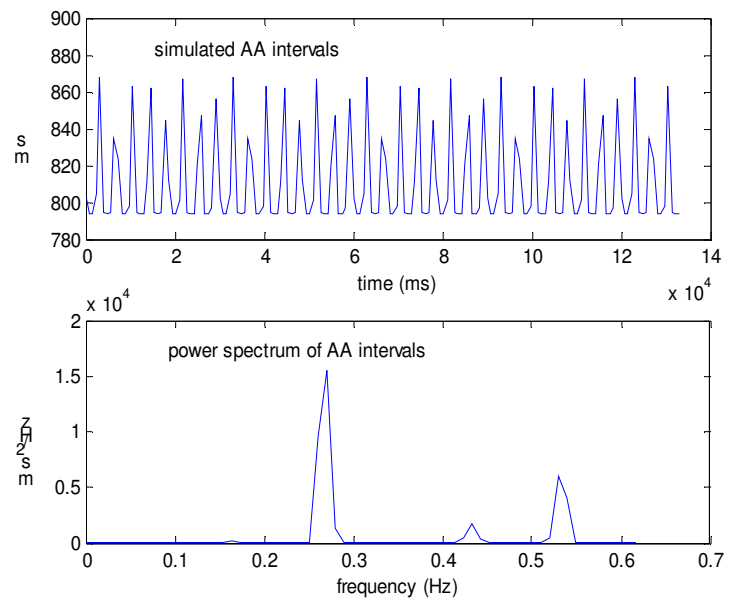

Figure 4. A simulated ARV and its power spectrum under $90 \mathrm{bpm}$ ventricular pacing obtained from the close-loop model. The mean atrial rate is $1.22 \mathrm{~Hz}$. The dominant frequency of ARV is $0.28 \mathrm{~Hz}$ that is consistent with the cardiac aliasing phenomenon.
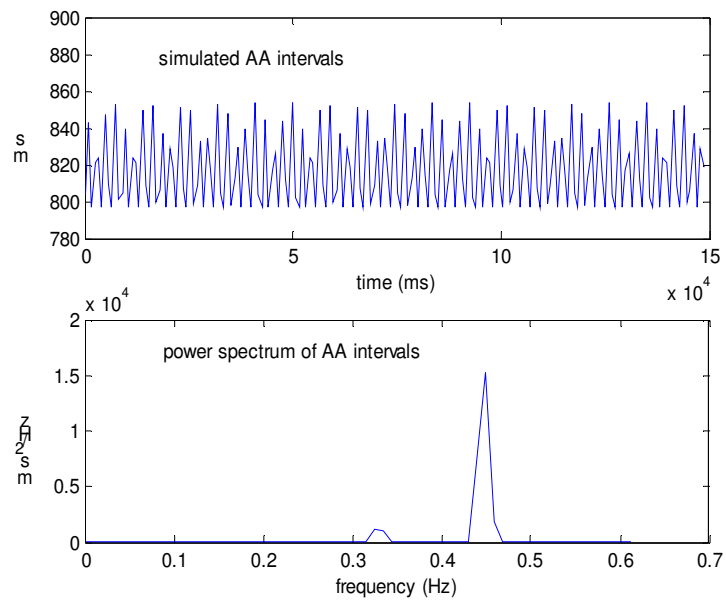

Figure 5. A simulated ARV and its power spectrum under $120 \mathrm{bpm}$ ventricular pacing obtained from the close-loop model. The mean atrial rate is $1.22 \mathrm{~Hz}$. The dominant frequency of $\mathrm{ARV}$ is $0.44 \mathrm{~Hz}$ that is consistent with the cardiac aliasing phenomenon.

\section{Discussion and conclusions}

The ARV in AV dissociation situation demonstrated a special pattern. It is found that the dominant frequency of ARV is an aliasing of ventricular pacing rate while considering the mean atrial rate as the sampling rate [6]. It is suggested that the BP pulse rate and waveform play an important role for this phenomenon. In this study, a model was created to test this supposition. The real BP waveform data was applied to simulate the ARV. The results showed that simulated ARV has consistent 
frequency feature with the real ARV. Moreover, a closeloop model automatically generating ARV and BP waveform data considering the effects of AV delay and baroreflex was used to observe the interaction between ARV and BP. The results confirm that the atrial rate fluctuations mainly reflect the fluctuation of entire BP waveform induced by the pulse and the changing SBP response to AV delay.

It must be emphasized that the model used in this study is too simplified and is not a proper model to describe the detailed dynamics of heart rate control with baroreflex. It is just used to illustrate the frequency domain feature of HRV, so the time domain feature of the simulated data may not consist with the real data well as shown in figure 2 and 3. However, it is useful for explaining heart rate fluctuation phenomenon observed in AV dissociation.

\section{Acknowledgements}

This study was supported by the grant NSC 92-2213E-038-008 of National Science Council of Taiwan ROC.

\section{References}

[1] Chiu HW, Kao T, Hsiao HC, Kong CW. Atrail Rate Variations in Atrioventricular Block Patients: Influences of Ventricular Rate and Atrioventricular delay. Computers in Cardiology 1999; 26:555-557.

[2] Robinson JL, Farr WC, Grupp G. Atrial rate response to ventricular pacing in the unanesthetized A-V blocked dog. Am J Physiol 1973, 224: 40-45.

[3] Simantirakis E, Skalidis E, Parthenakis F, Chrysostomakis S, Manios E, Kochiadakis G, Vardas P. Impact of atrioventricular delay on heart rate variability of paced patients with and without heart failure. Int J Cardiol 1995, 52: $235-239$.
[4] Taylor JA, Eckberg DL. Fundamental relations between short-term RR interval and arterial pressure oscillations in humans. Circulation 1996, 93: 1527-1532.

[5] Laitinen T, Hartikainen J, Niskanen L, Geelen G, Lansimies E. Sympathovagal balance is major determinant of short-term blood pressure variability in healthy subjects. Am J Physiol 1999, 276: H1245-H1252.

[6] Chiu HW, Kao T, Hsiao HC, Kong CW. Cardiac aliasing in atrial rate variation response to fixed-rate ventricular pacing. Computer in Cardiology 2001, 28:505-508.

[7] Rother M, Witte H, Zwiener U, Eiselt M, Fisher P. Cardiac aliasing - a possible cause of misinterpreting cardiorespirographic data in neonates. Early hum Developm 1989, 20: 1-20.

[8] Zwiener U, Luthke B, Bauer R, Richter A, Hoyer D. Forms of physiological aliasing within the heart rate fluctuations by higher frequent respiratory movements. J Physiol Pharmacol 1994, 45: 563-572

[9] De Boer RW, Karemaker JM, Strackee J. Hemodynamic fluctuations and baroreflex sensitivity in humans: a beat-tobeat model. Am J Physiol 1987, 253: H680-H689.

[10] De Boer RW, Karemaker JM, Strackee J. Spectrum of a series of point events, generate by the integral pulse frequency modulation model. Med Biol Eng Comput 1985, 23: $138-142$.

[11] Sanderson AC. Input-output analysis of an IPFM neural model: effects of spike regularity and record length. IEEE Trans Biomed Eng 1980, 27: 120-131.

[12] Type your references here. With the reference style, numbering is supplied automatically.

Address for correspondence

Hung-Wen Chiu

Graduate Institute of Medical Informatics, Taipei Medical University, $250 \mathrm{Wu}$-Hsing Street, Taipei 110, Taiwan.

hwchiu@tmu.edu.tw 